qualified by personal attendance to issue a death certificate.

The new proposals would end all this because they simply require the practitioner to state the cause of death if he knows it or, if he does not, to say so. If he has attended during the seven days preceding death he can issue a certificate direct to the registrar; if he has not, but nevertheless knows the cause of death, he can inform the coroner of his views and of the reasons for them. The coroner can then himself issue a certificate based on the clinical cause of death or order a necropsy, according to circumstances and the strength of the evidence.

A point which Professor Knight omits, and which seems pertinent, is that the form of certificate recommended by the Brodrick Committee (fig 2 in their report) would set out the possible reasons for notifying the coroner, and this would enable the practitioner to check that he was entitled to sign the certificate. There would be an increase in notifications to the coroner, but not all would require necropsy. In spite of the limitations of clinical diagnosis in relation to some conditions, radiodiagnosis, ultrasound, endoscopy, electrocardiography, and chemical pathology are all playing an increasingly important part in confirming the clinical picture, and one would expect coroners to rely more in the future on a well-documented diagnosis during life than the law has permitted them to do in the past.

To me the new proposals represent a logical singleminded approach to the problems of certifying the cause of death, and by imposing on the doctor the legal duty of notifying the coroner in appropriate cases they relieve him of the unpleasant choice, which he now occasionally has, between following the wishes of the bereaved relatives and following his inner conviction that he really does not know why the deceased has died.

Medicolegal Centre,

H H Pilling

\section{Avoidance of tracheostomy in sleep apnoea syndrome}

SIR,-Dr J R Stradling's report on the treatment of sleep apnoea syndrome (7 August, p 407) appears to imply that tracheostomy is the routine treatment of choice in this condition and that more minor surgical procedures are an innovation in its therapy. I should like to point out that this is not the situation at all.

Obstructive apnoea during sleep arises due to an obstruction to airflow within the respiratory tract, the usual area affected being the pharynx due to incoordination of the pharyngeal musculature (although studies reporting varying sites of obstruction have been published). It has been proposed that the underlying lesion may reside in the area of the tractus solitarius of the hindbrain. ${ }^{1}$ Many people with this predisposition are probably asymptomatic, only displaying symptoms when there is further obstruction to their compromised respiratory tract-for example, tonsillar hypertrophy.

The first therapeutic attempts are usually aimed at removing such aggravating factors, followed, if unsuccessful, by medical treatment. ${ }^{2}$ This normally consists of protriptyline, which is thought to improve airway co-ordination, ${ }^{3}$ while medroxyprogesterone is used in cases of central apnoea (lack of respiratory drive) as a respiratory stimulant. Tracheostomy, being such a major procedure, is held back until there is no alternative: "extremely severe sleep apnoea due to repetitive upper airway obstruction constitutes an urgent indication for tracheostomy in the absence of correctable causes," 2 or where the syndrome has produced life-threatening cardiovascular sequelae.

Casualty Department,
Cardiff Royal Infirmary, Cardiff

Chokroverty S, Sharp JT. F Neurol Neurosurg Psychiat 1981;44:970-82.

Clark RW. Primary care 1979;6:653-79.

Coaker LA, Quan SF. Ariz Med 1981;38:446-50 Med 1980;26:347-72.
Milleminault C, Cummey Jement WC. Adv Int

SIR,-Dr J R Stradling reported a case of sleep apnoea syndrome in a 37-year-old man which resolved after tonsillectomy and submucous resection (7 August, $p$ 407). This condition is also seen in children and I report here the case of a 6-year-old obese boy who also made a dramatic improvement after tonsillectomy.

The child was seen at the age of $2 \frac{1}{2}$ years in the outpatient department because of obesity. His weight was well above the 97th centile, and he was advised to go on a diet. He had an adenoidectomy at $4 \frac{1}{2}$ years because of nasal obstruction and speech delay. Between January and May 1982 he had three admissions with acute respiratory difficulties occurring while asleep in the early morning. In each of these he had evidence of pulmonary consolidation and upper airway obstruction. On one occasion he had an examination under anaesthetic of his upper airway-this was normal and large tonsils were noted. On each occasion he made a dramatic recovery within 12 hours. Investigations for a cause of recurren chest infections were negative. There was anxiety about two possible diagnoses: ( $a$ ) recurrent acute hypostatic pneumonia from obesity hypoventilation (although a resting $\mathrm{PCO}_{2}$ had always been normal) and (b) intermittent severe upper airway obstruction secondary to tonsillar enlargement-that is, sleep apnoea syndrome. Although he had never had daytime hypersomnolence, he had always snored.

He was considered for tonsillectomy, and ear oximetry studies were performed. During one overnight recording obstructive apnoea produced swings of arterial oxygen saturation from $96 \%$ to $65 \%$ on five occasions. Tonsillectomy was performed uneventfully. Further overnight oxygen saturation recordings wer made five days later and there were no swing in oxygen saturation. He has not snored since the operation and has had no further admissions.

\section{Department of Child Health}

London SW17 $0 Q T$ T

SIR,-I was interested to read Dr J R Stradling's report of this condition (7 August, p 407). Some 10 years ago I picked up such a case. The man had choking attacks at night and went quite blue. He was forced to sit on the edge of his bed, and his wife banged him on the back. His breathing then returned to normal. The precipitating factor was when he lay on his back. I had not met the problem before, and I could not name it. I explained it to myself as being similar to when the tongue of an anaesthetised patient falls back and obstructs his breathing. I applied an old country method said to stop a man from snoring. I gave the patient a roll of elastoplast and a wooden cotton bobbin. He got his wife to strap this in the centre of his back, so that it was virtually impossible for him to sleep on his back. After this had been applied his attacks ceased. I have been retired for almost eight years, and I gather my ex-patient still applies the bobbin every night. It is perhaps worth bearing this method in mind as a first-aid measure for a very alarming syndrome.

Ashby de la Zouch

C A H WatTS

Leicestershire LE6 5 GZ

SIR,-Dr J R Stradling's short report (7 August, p 407) on the avoidance of tracheostomy in sleep apnoea was both educational and interesting. However, we would like to make two comments.

Firstly, most patients with this syndrome do not have a surgically treatable obstruction and therefore tracheostomy remains the primary form of surgical management. The surgically treatable obstructions that have been described include such pathologies as micrognathia, macroglossia (in acromegaly), vocal cord paralysis, glottic webbs, and tracheal stenoses. The additional obstructive problem of tonsils and adenoids tends to be more of a problem in children and here surgical intervention is usually of great benefit. Many patients have other reversible factors and advice to cease night sedation (mistakenly taken to help improve sleep), to abstain from alcohol, and to reduce weight may help. Similarly, treatment of any conditions causing tissue swelling or oedema such as hypothyroidism or nephrotic syndrome may be useful. However, this patient also had obstruction in the form of asthma, and recent evidence has pointed to abnormalities in the movement and tone of the upper airways in patients with active lower airways obstruction. Therefore could vigorous anti-asthmatic therapy have had any effect on this patient's condition?

Secondly, the terms Pickwinian syndrome, as used in Dr Stradling's report, and Ondine's curse should no longer be used when describing sleep apnoea. The Pickwinian syndrome was originally used to describe daytime somnolence and cor pulmonale in obese patients and was wrongly attributed to daytime hypoventilation and $\mathrm{CO}_{2}$ retention. It is now well accepted that these patients have nocturnal sleep apnoea which leads to daytime tiredness. However, since sleep apnoea can be obstructive or central or a combination of both and since these entities can occur in either obese or non-obese subjects it can only be misleading to allude to the Pickwinian syndrome. When discussing this group of patients it would seem wiser to only use the terms obstructive and/or central apnoea and then list the numerous secondary contributing factors that may exist in each patient. The use of eponyms in this condition serves only to perpetuate myths and confuse the reading public.

\section{P J THOMPSON} E SAWICKa

Cardiothoracic Institute,

Brompton Hospital

' Sukerman S, Healey GB. Laryngoscope 1979;89:

878-85.
Guilleminault C, Eldridge F. Pediatrics 1976;58:23-30. Collet P Brancatisan Te Engel L. Aust NZ 3 Med $1982 ; 12: 221$.

Burwell C, Rabin E, Whaley R, Biddman A. $A m \mathcal{f}$ Med $1956 ; 21: 811-8$. 